\title{
HYBRID REALISATION OF HIGH PRECISION ANALOG CONVERSION MODULES
}

\author{
L. KUN \\ Industrial Research Institute for Electronics HIKI Budapest, Hungary \\ (Received October 1977)
}

\begin{abstract}
High precision analog modules are manufactured using film technologies. Components which are critical as regards operation and stability are made by thin film technology, additional elements and interconnection of active components are made using thick film technology.

Using these two separate manufacturing methods to obtain high stability and accuracy, optimum cost and yield can be achieved. To illustrate these facts, two circuits are discussed in detail, a voltage to frequency converter and a log amplifier.
\end{abstract}

\section{INTRODUCTION}

In recent years the use of hybrid integration in the realisation of analog conversion modules has increased. Here, two particular circuits are described. The primary uses of voltage to frequency converters are in control technology and transmission systems. The log modules are used in measurement and control systems.

The two circuits discussed here were designed to meet the industrial requirements of low cost and high yield. Because of this aspect, designs were preferred in which the parameter spread of monolithic devices is negligible and the transfer factor is trimmed by a single resistor.

\section{VOLTAGE TO FREQUENCY CONVERTER}

Modular V-F converters provide inherently monotonic A/D conversion. Their pulse rate output may be transmitted over 2 wire lines. Current and voltage input types are available. Applications include A/D conversion, use with a counter as a 3.5 digit DVM or analog data recording and linear phase lock loops.

\subsection{Basic Operation}

One of the best methods of obtaining high precision voltage to frequency conversion is the charge equalising method. Referring to Figure 1, the voltage $V_{c}$ across $C$ decays when $k=0$. When $V_{c}$ reaches zero, $K_{1}$ changes state, $k$ is now logical 1 for time $t_{0}$. The change in charge on $C$, due to $I_{s}$ is given by:

$$
\Delta Q=\left(I_{s}-I_{\text {in }}\right) t_{0}
$$

The voltage $V_{c}^{\prime}$ on $C$ at the end of $t_{0}$ is:

$$
V_{c}^{\prime}=\left(I_{s}-I_{\text {in }}\right) t_{0} / C
$$

At the end of $t_{0}, k=0$ and the voltage on $C$, due to $I_{\text {in }}$, decreases. The time taken by $V_{c}$ to reach the comparison level is:

$$
t_{1}=\left(I_{s}-I_{\text {in }}\right) t_{0} / I_{\text {in }}
$$

The total period is $t_{0}+t_{1}$ and output frequency is given by:

$$
f_{\text {out }}=I_{\text {in }} / I_{s} t_{0}
$$

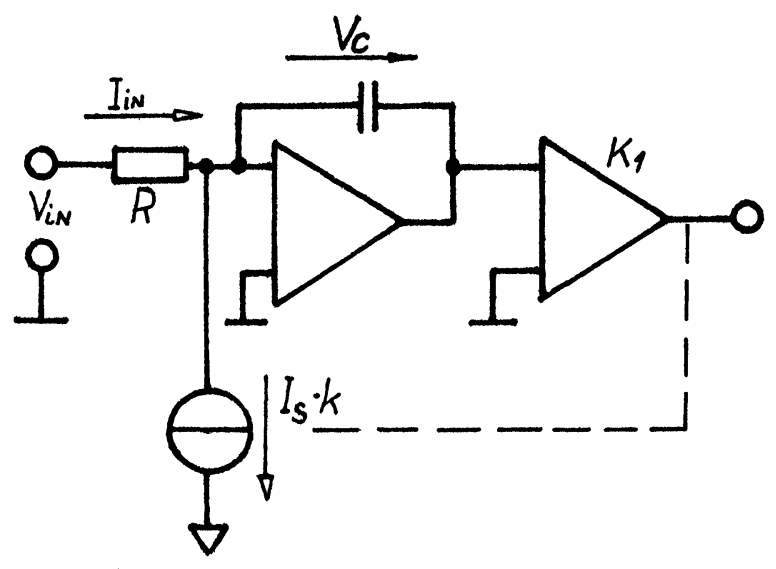

FIGURE 1 Basic charge equalising method. 


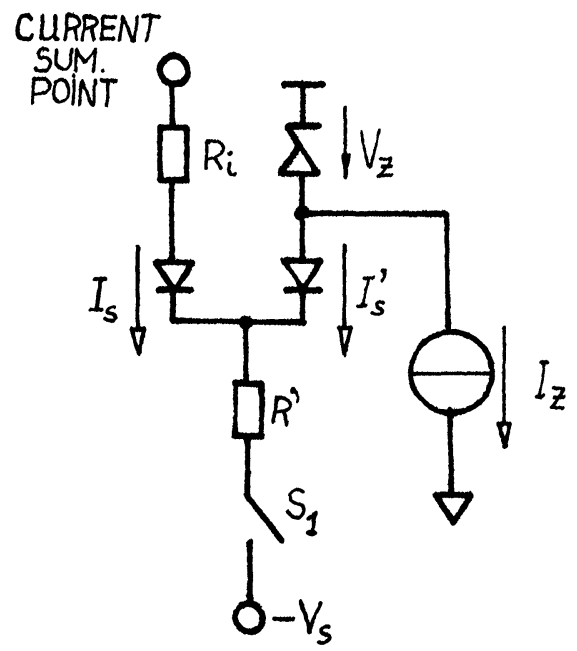

FIGURE 2 Realisation of switched current source.

Since current generator $I_{s}$ is connected to the inverting input of the op. amp. (virtual ground), it can be realised by the circuit shown in Figure 2. With switch $S_{1}$ open, currents $I_{s}$ and $I_{s}^{\prime}$ are zero, because the diodes are reverse biased. When switch $S_{1}$ is closed, the diodes will be forward biased. Since the diodes are realised by a double diode, the voltage drops at each diode are equal if the currents through the diodes are equal. This may be carried out by careful selection of $R^{\prime}$. The current flowing into the virtual ground is $V_{z} / R_{i}$. Switch $S_{1}$ is closed during time $t_{0}$. To generate a high precision time reference, the circuit in Figure 3 is used. The time from opening switch $S_{2}$ until the comparator changes its state, is:

$$
t_{0}=R_{M} C_{M} \ln \left(R_{1} / R_{2}+1\right)
$$

Substituting Eq. (4) and the value of $I_{s}$ into Eq. (3), the output frequency is:

$$
f_{\text {out }}=I_{\mathrm{in}} R_{\mathrm{i}} / R_{M} C_{M} V_{z} \ln \left(R_{1} / R_{2}+1\right)
$$

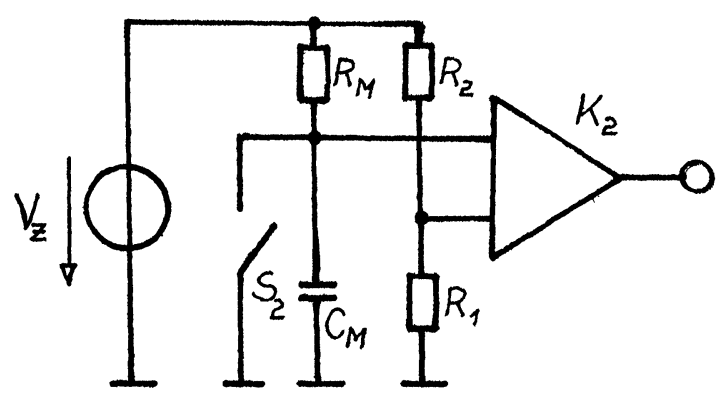

FIGURE 3 Simplified time constant circuit $\left(t_{0}\right)$.
Besides the passive elements in Eq. (5), the accuracy of conversion is influenced by the switching properties of $S_{1}$ and $S_{2}$, and the stability of comparator $K_{2}$. The only requirement for comparator $K_{1}$ is the short term stability of level of comparison.

\subsection{Choice of Technology}

From Eq. (5) it is clear that output frequency, in the case of current control, depends on resistor ratios, and absolute values of $V_{z}$ and $C_{M}$. Substituting $V_{\text {in }} / R$ into $I_{\mathrm{in}}$, for the case of voltage control, we find that only resistor ratios $R / R_{i}, R_{1} / R_{2}$ and time constant $R_{M} C_{M}$ determine the frequency. To obtain high precision current and voltage control, resistors with low TCR, NPO capacitors and a precise voltage reference must be used. To get the lowest TCR, we use thin film resistors in critical places of operation. The circuit was divided intc two parts. One part is the high precision thin film resistor network, the other is the thick film part, which contains encapsulated, active elements, the capacitor $C_{M}$ and resistors which are not critical in respect of operation. We use glass substrate for thin film resistors. The sheet resistance is $200 \Omega / \mathrm{sq}$. in order to obtain the highest stability ( $50 \mathrm{ppm} / \mathrm{year}$ ) and low TCR $\left(0 \pm 25 \mathrm{ppm} /{ }^{\circ} \mathrm{C}\right)$. It is important that the temperature tracking between resistors is better than $5 \mathrm{ppm}$. For this purpose, nickel-chromium was selected as the film, since it exhibits the best stability of electrical properties. The $\mathrm{NiCr}$ is vacuum deposited. A photolithographic masking operation is then performed on a wafer which delineates the resistor and conductor paths.

Besides a thin film network, we use thick film technology to make interconnections between active circuits and thick film resistors for additional functions such as controlling switches, biasing and offset nulling op. amps.

The thick film circuit function is controlled with etalon resistors in place of thin film resistors. After these processes, two parts are built together. Terminal pins are used to fix the two parts. Terminal pins surround the ceramic substrate of the thick film and glass substrate of thin film. (Figure 5 shows the cross-sectional view).

\subsection{Final-Trimming Process}

Capacitor $C_{M}$ and Zener-diode $V_{z}$ have a relatively high tolerance spread, and hence the circuits have to be trimmed functionally to match the design parameters. From Eq. (5), $f_{\text {out }} / 10,00 \mathrm{~V}>10,00 \mathrm{kHz}$ then $R$ is adjusted, otherwise $R_{i}$ is adjusted. Functional trimming is carried out by laser beam. 


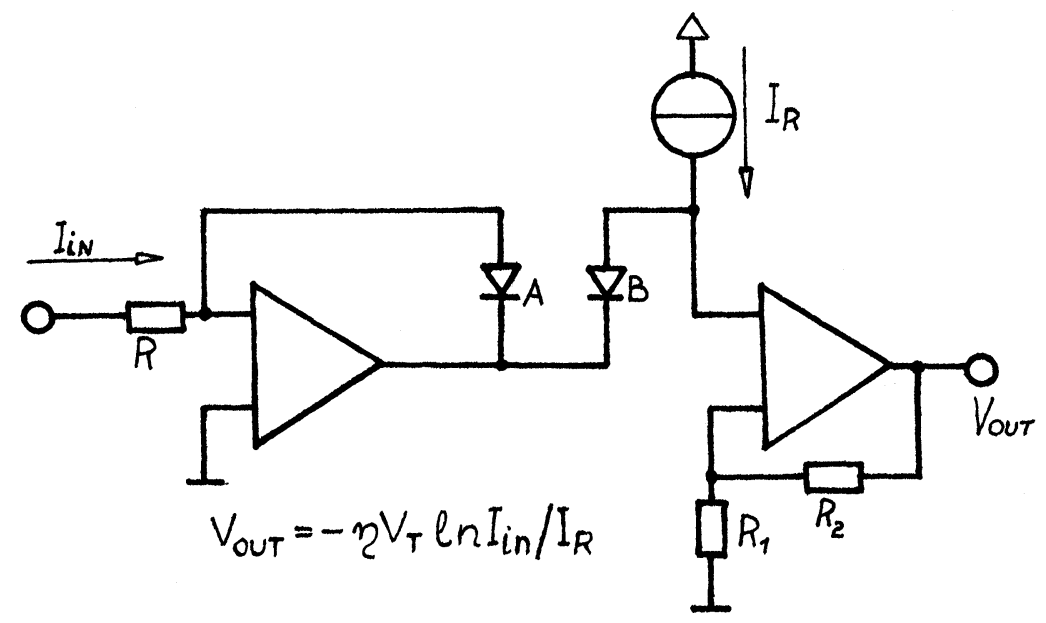

FIGURE 4 Basic log. circuit.

\section{LOGARITHMIC AMPLIFIER}

Another example illustrating the use of mixed technology is the log. amplifier. Such devices use the nonlinear volt-ampere relationship of the $p-n$ junction itself. Because dual transistors with good log. performances are not at present available for hybrid integration, the double diode was used as the log element.

\subsection{Double Diode Configuration}

The basics of operation are shown in Figure 4. The idealised transfer function (see Eq. (6)), has a temp- erature dependent term $\left(V_{T}\right)$. For compensation, a thermistor is used in place of $R_{1}$ in practical circuits. The TCR achieved is about $400 \mathrm{ppm} /{ }^{\circ} \mathrm{C}$. To achieve a better TCR, a hybrid "thermostat" is used.

\subsection{Heating the Log Element}

The constant current through side $B$ of the double diode generates a temperature dependent voltage drop. This voltage is measured and kept constant. The measured voltage is subtracted from a reference voltage and amplified to control the heating element. We chose a medium power transistor to perform the

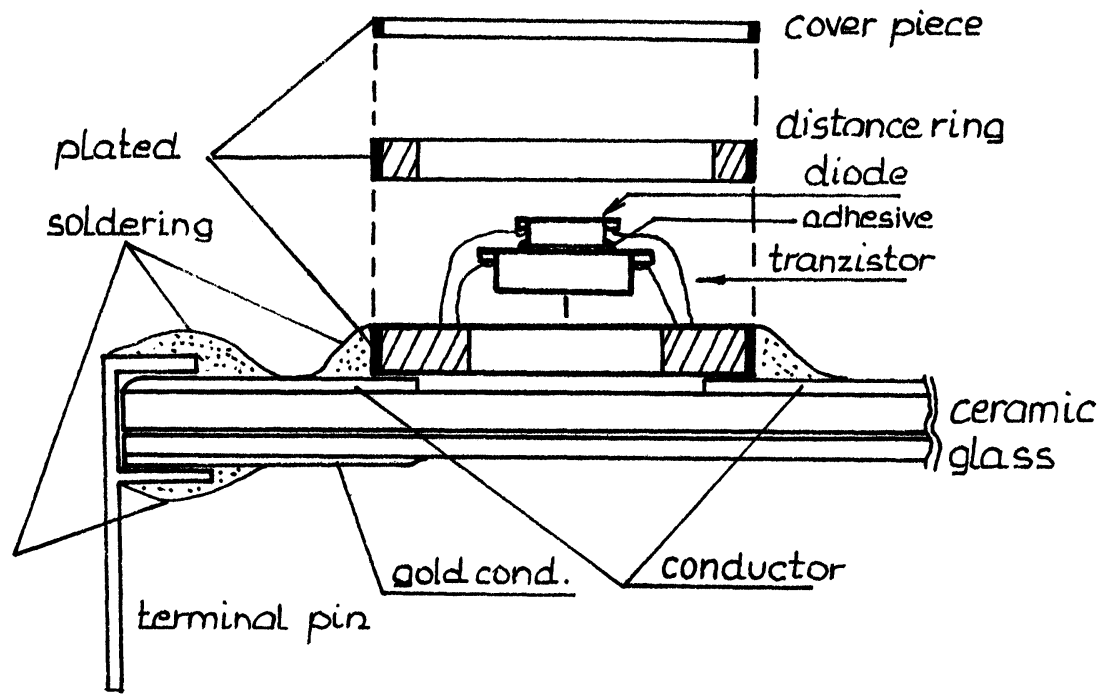

FIGURE 5 Cross-sectional view of log. circuit. 


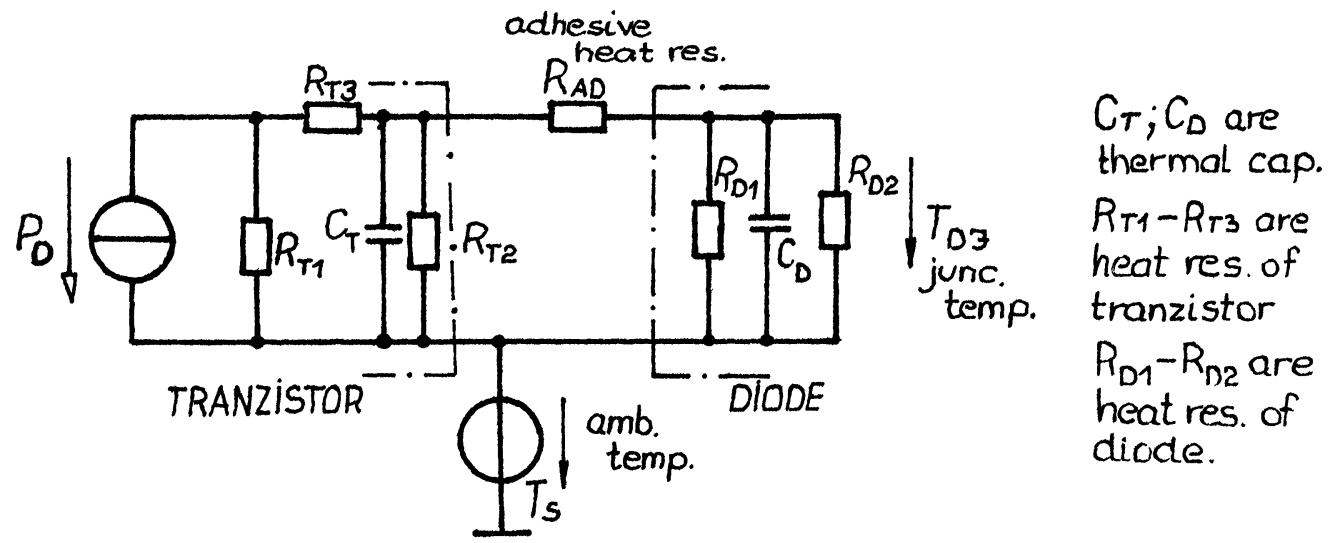

FIGURE 6 Thermal model of thermostat.

heating function. The transistor and diode are kept in close thermal contact by means of adhesion. This pair is mounted into a frame, shown in Figure 5. Six terminals were plated on the two opposite sides of the frame, to make mounting of the ceramic substrate by soldering possible. The equivalent circuit for thermal behaviour is shown in Figure 6. The absolute temperature of the diode junction must be a constant value. This temperature, $T_{J}$ is:

$$
T_{J}=T_{S}+K^{\prime} P
$$

where $K^{\prime}=T_{D J} / P \sim 200^{\circ} \mathrm{C} / \mathrm{W}$. This value was measured and agrees well with theory.

\subsection{Circuit Description}

Figure 7 shows an arrangement for keeping the diode junction temperature constant. An additional op. amp. is used to provide the control voltage for the heating element. The amplification factor for the voltage generated by $I_{R}$ is critical because of the two time constants in the control loop (see Figure 6). The amplification is limited to the 150 to 450 range. The time required to reach the final temperature is around 2 min. depending on ambient temperature. The temperature of the junction is approximately $90^{\circ} \mathrm{C}$. The stability of temperature is $1 \%$ in the operating range.

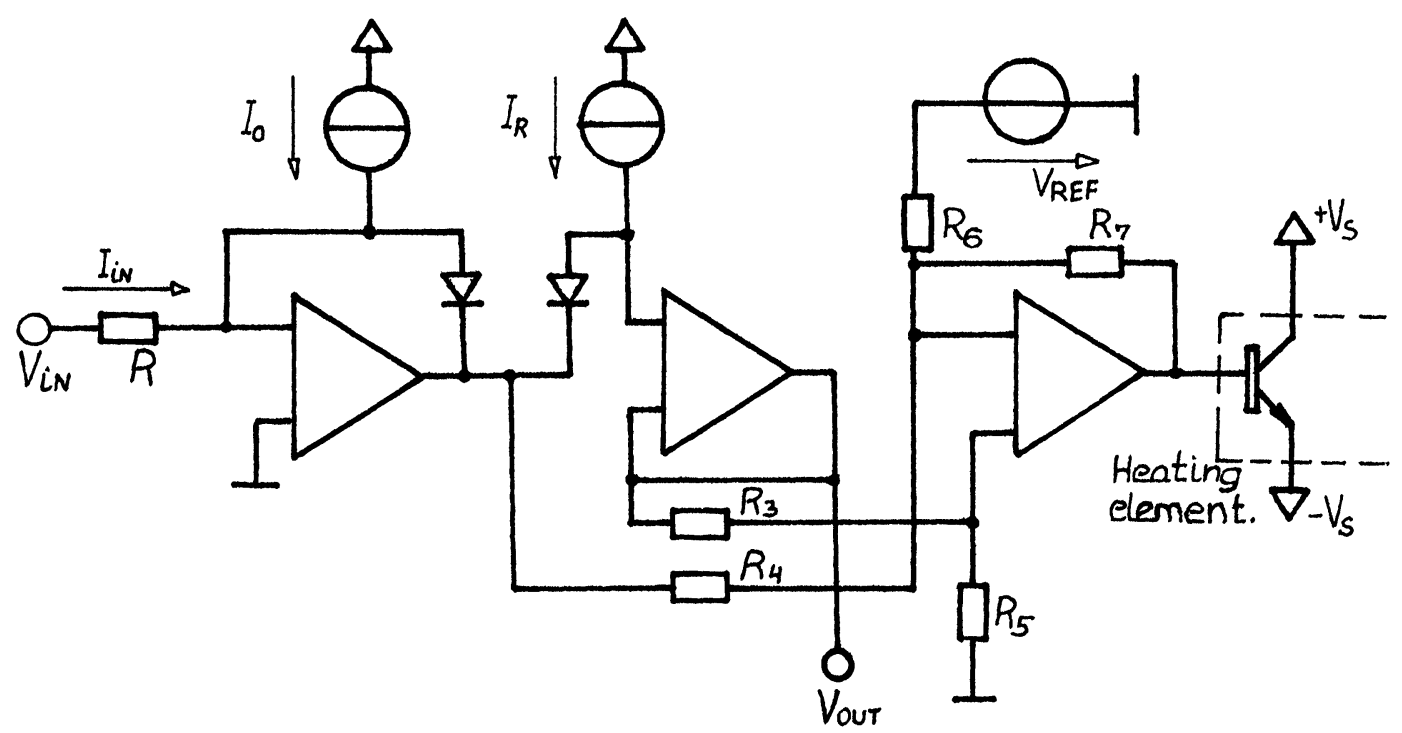

FIGURE 7 Final circuit diagram of log. circuit. 
The current $I_{0}$ in Figure 7 provides compensation for the reverse saturation current of the diodes which is significantly increased due to the high junction temperature.

\subsection{Construction}

When manufacturing the circuit, the same technological steps were used as described for the V-F converter. Resistors, whose stability and TCR are critical, are made by thin film technology. Other resistors, used for offset nulling for op. amps., $I_{0}$ correction, bulk resistance correction of diodes etc., are manufactured from thick film material.

\subsection{Trimming Procedure}

After circuit function monitoring, active trimming is performed by air abrasive methods to adjust offset voltages of op. amps. The two parts are built together and $I_{R}$ is modified to get zero output voltage, when applying $1,000 \mathrm{~V}$ to the input. (Resistors, made from thin film, are adjusted to the nominal value with required accuracy.)

The resistor $I_{R}$ is divided into two parts. $90 \%$ of nominal value is manufactured by thin film technology and the other part by thick film. The thick film part is adjusted actively to obtain the desired transfer function.

\section{CONCLUSION}

High precision modules can be realised by careful selection and processing of passive elements. Mixed technology gives high productivity, small size and high reliability. Thin film resistors promise low TCR and high stability, whilst thick film gives high reliability connections amongst active elements.

Active trimming procedures eliminate selection of components such as $C_{M}$ and $V_{z}$. Offset trimming procedures enable the use of low cost op. amps. in critical areas. 

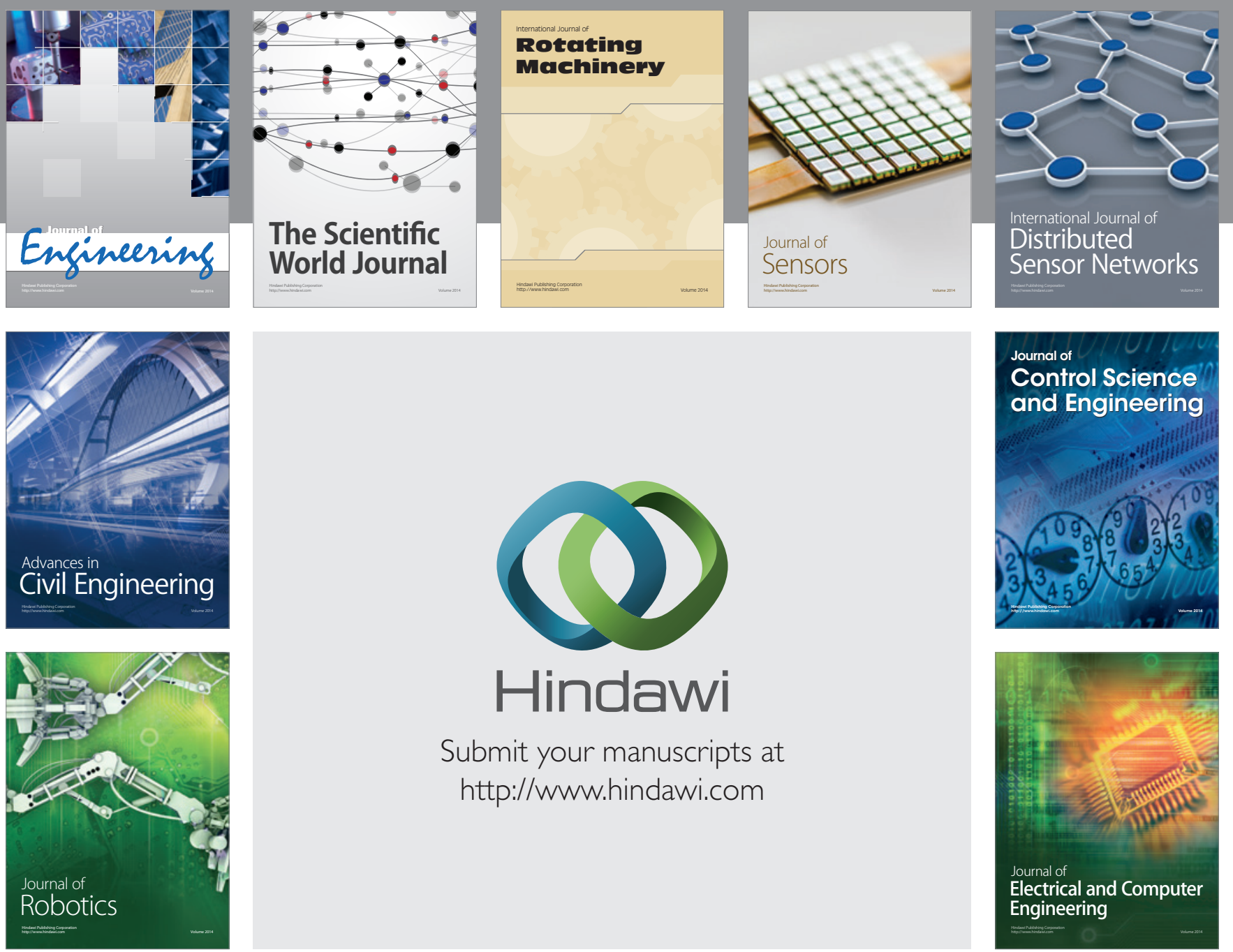

Submit your manuscripts at

http://www.hindawi.com
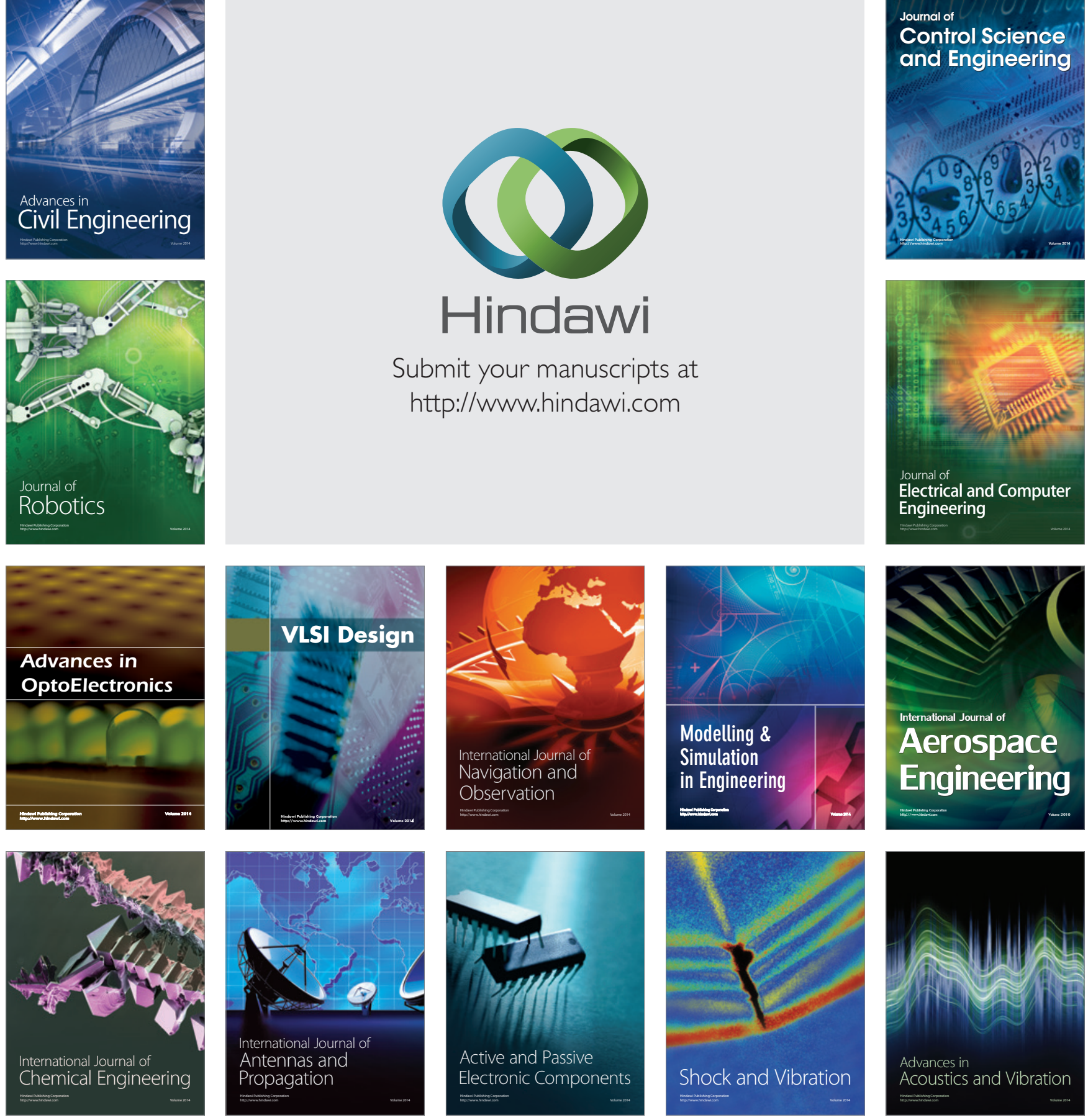